\title{
All-nanocellulose nonvolatile resistive memory
}

\author{
Umberto Celano ${ }^{1,2,5}$, Kazuki Nagashima ${ }^{3,5}$, Hirotaka Koga ${ }^{4}$, Masaya Nogi ${ }^{4}$, Fuwei Zhuge ${ }^{3}$, Gang Meng ${ }^{3}$, \\ Yong $\mathrm{He}^{3}$, Jo De Boeck ${ }^{1}$, Malgorzata Jurczak ${ }^{1}$, Wilfried Vandervorst ${ }^{1,2}$ and Takeshi Yanagida ${ }^{3}$
}

Single-use disposable nonvolatile memory devices hold promise for novel applications in internet of everything (loE) technology by storing the health status of individual humans in daily life. However, conventional memory devices are not disposable because they are mostly composed of non-renewable, non-biodegradable and sometimes toxic materials, causing serious damage to ecological systems when they are released to the environment. Here, we demonstrate an environment-friendly, disposable nonvolatile memory device composed of 99.3 vol.\% nanocellulose. Our memory device consists of a nanocellulose-based resistive-switching layer and a nanopaper substrate. The device exhibited nonvolatile resistive switching with the capability of multilevel storage and potential scalability down to the single nanofiber level (ca. $15 \mathrm{~nm}$ ). The biodegradability of our memory device was confirmed by burying it in natural soil for 26 days.

NPG Asia Materials (2016) 8, e310; doi:10.1038/am.2016.144; published online 16 September 2016

\section{INTRODUCTION}

Recently, the internet of everything (IoE), which enables information transfer between individual humans, animals, things and places through the internet environment, has attracted much attention as a novel electronic technology next to the internet of things. ${ }^{1-3}$ IoE technology offers real-time monitoring and analysis of the health status of individual humans in daily life. However, some data cannot be obtained if the target temporarily leaves the internet environment. Light-weight, single-use and disposable nonvolatile memory devices hold promise for novel applications in IoE technology because they can store these data temporarily when the target enters a noninternet environment, and they can be disposed of when they complete this function. Conventional memory devices are not disposable because they are mostly made of non-renewable, non-biodegradable and sometimes toxic materials, causing serious damage to ecological systems when they are emitted to the environment. ${ }^{4}$ Therefore, the development of nonvolatile memory using renewable, biodegradable, nontoxic and environment-friendly materials is strongly required for disposable IoE electronic devices.

A paper-based memory device is one of the most promising options because cellulose is the most abundant renewable material. Among paper-based electronics, devices using so-called nanopaper have recently attracted increasing attention. Nanopaper is made of nanocellulose, for example, cellulose nanofibers or cellulose nanorods obtained by mechanically fibrillated wood pulp. Nanopaper has foldability, a relatively high resistance to water and solvents, surface flatness and high optical transparency. ${ }^{5-9}$ Furthermore, nanopaper has an extremely low thermal expansion coefficient $\left(6 \times 10^{-6} / \mathrm{K}\right)$ compared with other polymer materials that are used for biodegradable electronics. ${ }^{10-16}$ Therefore, nanopaper has recently received much attention as a substrate for flexible electronics. ${ }^{9,17-29}$ To date, various electronic devices including organic/inorganic thin-film transistors, ${ }^{9,21-23}$ light-emitting diodes, ${ }^{24}$ solar cells, ${ }^{23,25,26}$ antennas ${ }^{27,28}$ and triboelectric generators ${ }^{29}$ have been demonstrated on nanopaper substrates. However, a nonvolatile memory, which is the essential component for IoE technology, has not been demonstrated on a nanopaper substrate so far. Here, we demonstrate an environment-friendly, disposable nonvolatile memory device composed of 99.3 vol.\% nanocellulose. Our memory device consists of a nanocellulose-based resistive-switching layer and a nanopaper substrate. The device exhibited electrochemical redox-based nonvolatile resistive switching with the capability of multilevel storage and potential scalability down to the single nanofiber level (ca. $15 \mathrm{~nm}$ ). The disposability of our memory device was also demonstrated by examining the biodegradability of the device in natural soil.

\section{MATERIALS AND METHODS}

\section{Preparation of cellulose nanofibers and nanopaper}

Cellulose nanofibers with a width of ca. $15-20 \mathrm{~nm}$ were obtained from softwood chips (Cryptomeria japonica). ${ }^{5,8}$ Briefly, the softwood chips were treated with sodium chlorite and potassium hydroxide. Then, an aqueous suspension of the as-prepared wood pulp $(0.5 \mathrm{wt} \%, 2 \mathrm{l})$ was subjected to a high-pressure water-jet system (Star Burst, HJP-25005E, Sugino Machine, Japan) equipped with a ball-collision chamber. The pulp suspension was ejected from a nozzle with a diameter of $0.15 \mathrm{~mm}$ under a high pressure of $245 \mathrm{MPa}$ with 50 repetition cycles. For the preparation of nanopaper, the cellulose nanofiber suspension was cast onto an acrylic plate, oven-dried at $50{ }^{\circ} \mathrm{C}$ for 1 day and then peeled off from the plate. Nanopaper memory devices were

\footnotetext{
${ }^{1}$ imec, KU Leuven, Belgium; ${ }^{2}$ Department of Physics and Astronomy (IKS), KU Leuven, Belgium; ${ }^{3}$ Institute for Materials Chemistry and Engineering, Kyushu University, Fukuoka, Japan and ${ }^{4}$ The Institute of Scientific and Industrial Research, Osaka University, Osaka, Japan

${ }^{5}$ These authors contributed equally to this work.

Correspondence: Dr K Nagashima or Professor T Yanagida, Institute for Materials Chemistry and Engineering, Kyushu University, 6-1 Kasuga-Koen, Kasuga, Fukuoka 816-8580, Japan. E-mail: kazu-n@cm.kyushu-u.ac.jp or yanagida@cm.kyushu-u.ac.jp

Received 9 April 2016; revised 11 July 2016; accepted 28 July 2016
} 
a

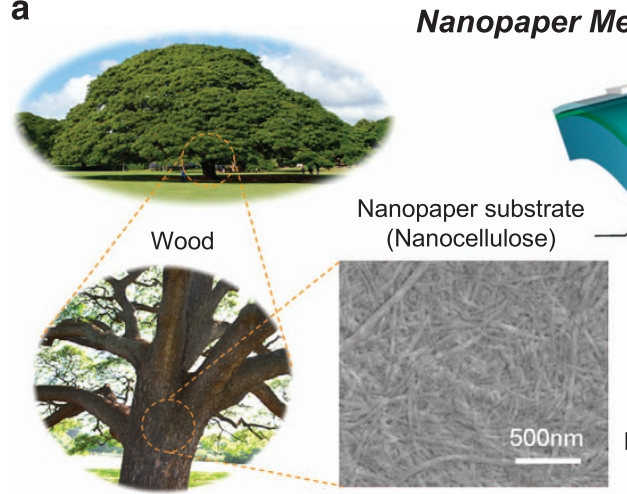

b

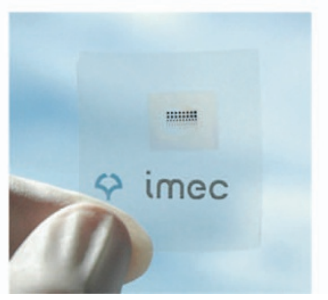

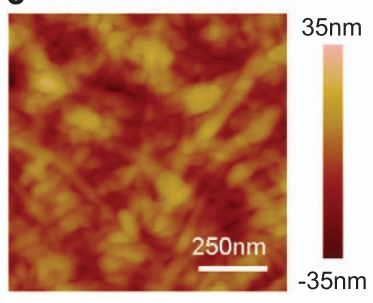

Ag electrode

Resistive-switching layer (Nanocellulose)
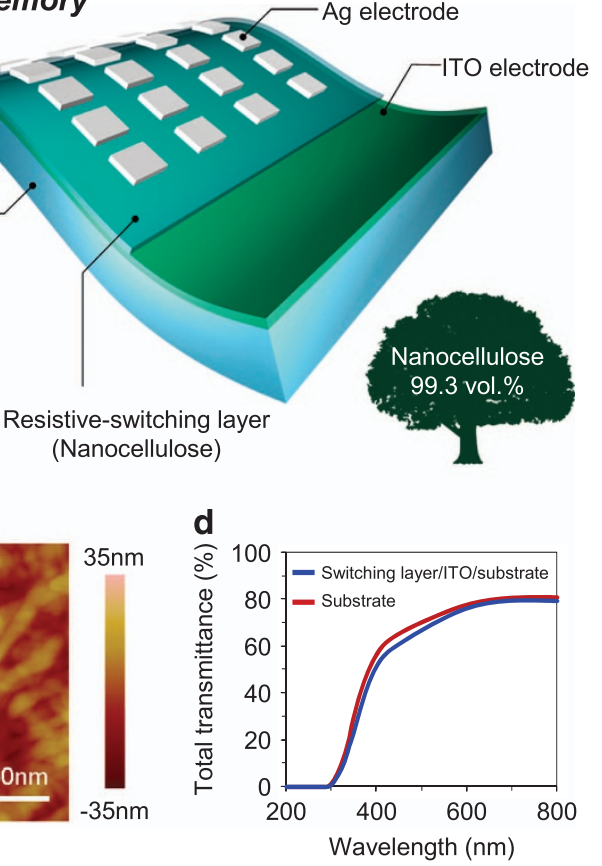

Figure 1 Structural information of a nanopaper memory device. (a) Schematic illustration of the constituent material of a nanopaper memory device. The nanopaper was made of softwood chips, and the field emission scanning electron microscopy (FESEM) image shows the surface morphology of the nanopaper substrate. The nanopaper memory device consists of 99.3 vol.\% nanocellulose. (b) Photograph of the nanopaper memory device. (c) Atomic force microscopy (AFM) image of the resistive-switching layer deposited onto an indium tin oxide (ITO)/nanopaper substrate. The thickness of the resistive-switching layer is ca. $100 \mathrm{~nm}$. (d) Absorption spectra of the nanopaper substrate and resistive-switching layer/ITO/nanopaper substrate. No significant change in transmittance was observed after the deposition of the ITO electrode and the resistive-switching layer.

fabricated using a thick nanopaper substrate $(\sim 30-50 \mu \mathrm{m})$. First, an indium tin oxide (ITO) bottom electrode with a thickness of $100 \mathrm{~nm}$ was deposited by radiofrequency sputtering at $1 \mathrm{~Pa}$ with $\mathrm{Ar} / \mathrm{O}_{2}$ mixed gas (100 p.p.m. $\mathrm{O}_{2}$ in $\mathrm{Ar}$ ). The deposition conditions were optimized to achieve good transparency and high conductivity. The resistivity of the layer was $\sim 3 \times 10^{-3} \Omega \mathrm{cm}$ that was measured by a standard four-probe measurement. Then, a resistive-switching layer was created by the drop-casting method, followed by the ITO deposition. For the deposition, we placed a droplet $(10 \mu \mathrm{l})$ of the cellulose nanofiber aqueous suspension $(0.02 \mathrm{wt} \%)$ on top of the ITO layer, followed by drying at $40^{\circ} \mathrm{C}$ for $15 \mathrm{~min}$. After the water evaporated, the thin cellulose nanofiber sheet was left on the ITO layer. The thickness of the resistive-switching layer was $100 \mathrm{~nm}$ that was controlled by the number of drop cycles, as shown in Supplementary Information S1. The thickness of the nanopaper was measured by a surface profiler. Three cycles of drop casting led to a layer thickness of $\sim 100 \mathrm{~nm}$. Finally, the $100 \mathrm{~nm}$-thick Ag top electrodes were deposited using radiofrequency sputtering and patterned by a metal mask. For the measurement of the total transmittance of the nanopaper, we used a ultraviolet-visible spectrometer with an integrating sphere (U-3900, Hitachi High-Tech., Tokyo, Japan). The measurement was performed using light in the wavelength range of 200-800 $\mathrm{nm}$. Atomic probe microscopy (AFM) was utilized to characterize the surface morphology of the nanopaper.

\section{Electrical transport measurement of nanopaper memory and cellulose nanofiber}

The current-voltage $(I-V)$ characteristics of the nanopaper memory devices were obtained using a semiconductor parameter analyzer (Keithley 4200-SCS, TFF Corporation, Tokyo, Japan). The experiments were carried out in atmospheric air conditions at room temperature. For all cases, the current compliance was employed to prevent the devices from destructive breakdown phenomena. For the single cellulose nanofiber measurement, we employed both a conductive-AFM (C-AFM) and a nanogap measurement. C-AFM was performed using a Pt-coated conductive cantilever. Before the measurement, the cellulose nanofiber aqueous solution $(0.02 \mathrm{wt} \%)$ was diluted to a 1:1000 ratio by deionized water and then dropped onto a $100 \mathrm{~nm}$ thick Ag-coated $\mathrm{Si}$ substrate. For the electrical measurement, the voltage was applied to the Ag electrode whereas the conductive cantilever was grounded. The measurement was performed in atmospheric air conditions at room temperature. On the other hand, the sample for the nanogap measurement was fabricated by $30 \mathrm{kV}$ electron beam (EB) lithography (JEM-7001F, JEOL, Tokyo, Japan and SPG-724 Nano printer, SANYU Electron, Tokyo, Japan). First, the contact electrode and marker pattern were patterned onto a $\mathrm{Si}$ substrate with a $100 \mathrm{~nm} \mathrm{SiO}{ }_{2}$ capping layer. The ZEP520A-7 (Zeon Corporation, Tokyo, Japan), which was used as an EB resist, was spin-coated at 5000 r.p.m. for 90 s, followed by baking at $180^{\circ} \mathrm{C}$ for $2 \mathrm{~min}$. After the EB lithography, the pattern was developed by immersion in ZED-N50 (Zeon Corporation) for $90 \mathrm{~s}$, and the Ti/Pt electrode was deposited by radiofrequency sputtering at $1 \mathrm{~Pa}$. Next, the diluted cellulose nanofiber aqueous suspension was dropped onto the pre-patterned substrate. After observing the single cellulose nanofiber by field emission scanning electron microscopy, the nanoelectrode pattern was overlaid by EB lithography. For making the contact to the cellulose nanofiber, Pt was utilized to avoid possible oxidization at the contact interface. The transport property of a single cellulose nanofiber was determined in vacuum conditions $\left(\sim 1 \times 10^{-3} \mathrm{~Pa}\right)$ to minimize the contribution of adsorbed water.

\section{Electrode removal and biodegradation experiments}

The removal of the electrode from the nanopaper memory was conducted in various solvents such as propanol, acetone, ethanol, $N, N$-dimethylformamide and water. Sonication was applied at $28 \mathrm{kHz}$ for $5 \mathrm{~min}$. After the sonication process, the residual electrode was evaluated. For the biodegradation experiment, we utilized natural soil. Then, 41 of soil was collected in September at 34:49:30 north latitude and 135:31:28 east longitude and kept in a plastic bag. The temperature and the humidity of soil were well controlled to be $22 \pm 2{ }^{\circ} \mathrm{C}$ 
and $92 \pm 3 \%$, respectively. As control experiments, nanopaper memory devices on a Si substrate and a polyethylene terephthalate substrate were also examined.

\section{RESULTS AND DISCUSSION}

Figure la shows the schematic image of a nanopaper-based memory device. Although our previous nanopaper-based nonvolatile memory device was fabricated on a Si substrate and/or $\mathrm{Al}$ foil, ${ }^{30}$ the nanopaper resistive-switching layer was created on a nanopaper substrate in this study. The thickness of the nanopaper substrate was $30 \mu \mathrm{m}$. A field emission scanning electron microscopy image showed the surface morphology of the nanopaper. A smooth surface with a densely packed nanofiber structure was found. Then, the $100 \mathrm{~nm}$ ITO thin film, which was used as an electrode, was sputtered onto the nanopaper substrate. The resistive-switching layer was created by drop-casting an aqueous solution of cellulose nanofibers. Although our previous study utilized Ag nanoparticle-decorated cellulose nanofibers for the resistive-switching layer, ${ }^{30}$ we utilized pure cellulose nanofibers in this study. Because the Ag nanoparticles have a large size distribution and are locally aggregated in the resistive-switching layer, it is in principle difficult for the Ag nanoparticle-containing nanopaper to obtain a uniform resistive-switching property when the device becomes smaller. On the other hand, our novel resistive-switching layer in this study is simply composed of cellulose nanofibers without Ag nanoparticles, allowing a uniform performance of resistive switching even when the device becomes smaller. The thickness of the resistive-switching layer was $100 \mathrm{~nm}$. This thickness was chosen to secure the electrical insulation and also to minimize the operating voltage for reaching the critical electric field of the initial forming process. Finally, a Ag electrode was patterned onto the resistiveswitching layer by a metal mask. A photograph of the fabricated nanopaper memory device is shown in Figure 1b. Remarkably, our nanopaper memory device has a $99.3 \%$ volume fraction of nanocellulose. This high-volume fraction was achieved by the fabrication of a thin conductive electrode layer on a flat surface nanopaper substrate that was unfeasible on regular paper. In general, a large part of semiconductor devices is composed of the substrate material. Therefore, the use of a nanopaper substrate plays a crucial role in obtaining such a high-volume fraction of nanocellulose. Although a paper substrate has previously been used for electronic device applications, no previous devices on regular paper have a cellulose volume fraction of $>50 \% .{ }^{31}$ This is because the surface roughness of regular paper is a few tens of micrometers, and therefore electronic devices on regular paper need an electrode of several tens of micrometer thickness to guarantee the continuity of the electrical conduction. Alternatively, the volume fraction of the electrode can be drastically reduced while maintaining its conduction property by using a flat surface nanopaper substrate. Figure 1c shows an AFM image of the resistive-switching layer/ITO/nanopaper substrate. The root-mean-square surface roughness of our nanopaper memory device was $7.41 \mathrm{~nm}$, and this is $2-3$ orders of magnitude smaller than that of the one on regular paper. Thus, the use of a flat surface nanopaper substrate plays a crucial role for obtaining the high-volume fraction of cellulose by reducing the volume of the electrodes. Note that in addition to the high-volume fraction of nanocellulose, our nanopaper memory device also has a high optical transparency. Because the cellulose nanofibers are laterally stabilized by hydrogen bonds and the interstices between the nanofibers are small enough, the light scattering of the nanopaper is drastically reduced and high optical transparency can be realized. ${ }^{5}$ Figure $1 \mathrm{~d}$ shows the total transmittance of the nanopaper memory as a function of the wavelength. The transmittance of the nanopaper was ca. $80 \%$ at a wavelength of $600 \mathrm{~nm}$, and this value was maintained even after depositing the ITO electrode and the resistive-switching layer. Thus, we successfully fabricated a nanopaper memory device that has a high-volume fraction of nanocellulose.

Next, we show the resistive-switching phenomena of the nanopaper memory device. Figure $2 \mathrm{a}$ shows the typical $I-V$ curves of the nanopaper memory. The measurement was performed at room temperature in atmospheric air conditions. In this measurement, a device size of $200 \times 200 \mu \mathrm{m}$ was used. The voltage was applied to the Ag top electrode, whereas the ITO bottom electrode was grounded. Initially, the device was highly insulative, and its resistance was $5.3 \times 10^{10} \Omega$ (purple line). An abrupt increase of current was observed when the voltage was increased to $1.5 \mathrm{~V}$. A current compliance of $1 \mathrm{~mA}$ was applied to prevent destructive electrical breakdown of the device. This is a so-called forming process that is used to initialize the resistive memory device. ${ }^{32-35}$ After the forming process, the device showed a low-resistance state (LRS), with a resistance of $63 \Omega$ (red line). The LRS was maintained until the negative voltage was applied. When the voltage reached $-0.5 \mathrm{~V}$, the current suddenly dropped, and the device showed a high-resistance state (HRS). This is the so-called RESET process. After the RESET process, the device showed a resistance of $3.3 \times 10^{9} \Omega$ (blue line). The LRS was seen again when a positive voltage of $1.0 \mathrm{~V}$ was applied. This is the so-called SET process. Note that the large ON/OFF memory window (a resistance ratio of over $10^{7}$ ) in our nanopaper memory is because of the highly insulative property of cellulose nanopaper (Supplementary Information S4). The LRS and the HRS were sequentially observed by the SET and RESET processes after initiating the device via the forming process. Thus, our nanopaper memory device showed a polarity-dependent bipolar resistive-switching behavior. ${ }^{32}$ Next, we performed retention measurements to examine the nonvolatility of the nanopaper memory device. Figure $2 b$ shows both the LRS and HRS resistances as a function of time. The voltage of $0.1 \mathrm{~V}$ was applied only when the resistances were read out. Although a slight variation in the HRS resistance was seen, both the HRS and LRS resistances were maintained for $10^{4} \mathrm{~s}$ while keeping a resistance ratio of over $10^{7}$, clearly indicating the nonvolatility of the nanopaper memory device. Thus, we successfully demonstrated nonvolatile resistive switching in our nanopaper memory device.

We further examined the multilevel memory effect of our nanopaper memory device. Because our nanopaper memory has a large ON/OFF memory window, the capability of the multilevel memory effect is expected by exploiting the intermediate resistance states in the memory window. The multilevel memory effect is an important feature for increasing the density of the data storage, as the memory density can be multiplicatively increased without increasing the device footprint. Figure $2 \mathrm{c}$ shows the LRS and HRS currents when we apply various compliance currents $(50 \mu \mathrm{A}, 500 \mu \mathrm{A}$ and $1 \mathrm{~mA})$ during the SET processes. The readout voltage was $0.1 \mathrm{~V}$. Three distinct LRSs appeared according to the levels of the compliance current. These states were maintained for $>2 \times 10^{3} \mathrm{~s}$, as shown in Figure $2 \mathrm{~d}$. Thus, we demonstrated the capability of multilevel memory in our nanopaper memory device. Next, we examined the scalability of the nanopaper memory device. Because the nanopaper was composed of a cellulose nanofiber network, the minimum unit of our memory device is a single cellulose nanofiber. Therefore, we performed a single cellulose nanofiber measurement. Figure 3a shows a schematic illustration of the single cellulose nanofiber measurement. The C-AFM was utilized for characterizing the transport property of a single cellulose nanofiber. The cellulose nanofibers were developed onto a $\mathrm{Ag}$ thin film-coated $\mathrm{Si}$ substrate. The details of the control of the number density of the cellulose nanofibers are provided in 

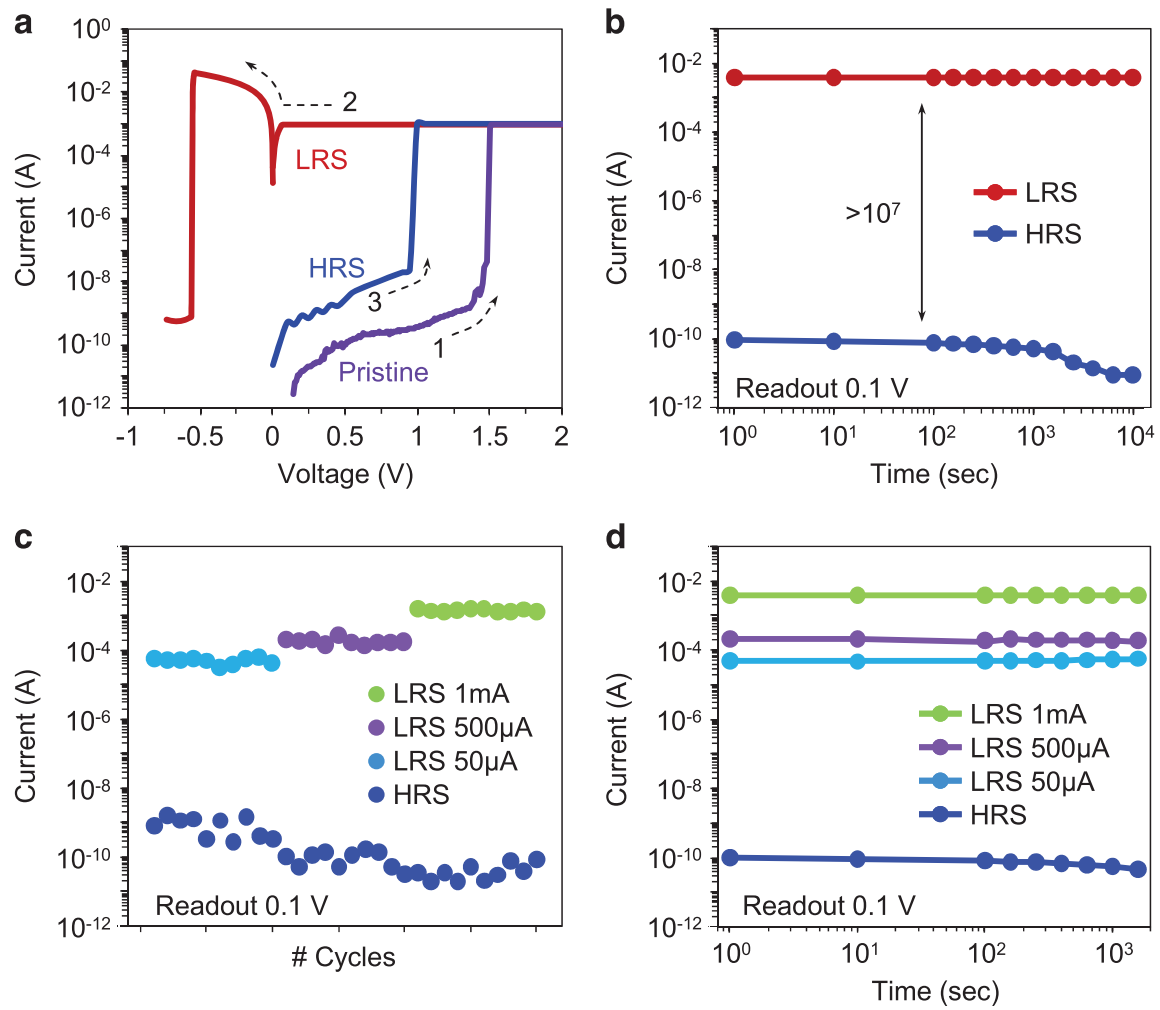

Figure 2 Electrical characterization of nanopaper memory. All of the measurements were performed in atmospheric air conditions at room temperature. (a) Current-voltage $(I-V$ curves of nanopaper memory devices. The device initially showed an insulative pristine state (purple). After the first soft breakdown process called the forming process, the device showed reversible resistance switching between the low-resistance state (LRS; red) and the high-resistance state (HRS; blue). (b) Retention characteristics for both the LRS and HRS of the nanopaper memory. The readout voltage was $0.1 \mathrm{~V}$. No significant degradation of the resistance state was observed for at least $10^{4} \mathrm{~s}$, with the resistance ratio maintained at $>10^{7}$, indicating the nonvolatility of the nanopaper memory device. (c) Multilevel memory effect of the nanopaper memory device. The compliance current was varied $(50 \mu \mathrm{A}, 500 \mu \mathrm{A}$ and $1 \mathrm{~mA})$ to create different LRSs. The readout voltage was $0.1 \mathrm{~V}$. Distinct LRSs were observed according to the range of the compliance current. (d) Retention characteristics of resistance states for multilevel operation. The readout voltage was $0.1 \mathrm{~V}$.

Supplementary Information S2. A Pt-coated cantilever was used as an electrode. Figure $3 \mathrm{~b}$ shows a topographic AFM image of the cellulose nanofibers. The maximum height of the cellulose nanofibers was ca. $15-20 \mathrm{~nm}$, consistent with the width of a cellulose nanofiber. ${ }^{36}$ The insulative property of the cellulose nanofiber was confirmed by the C-AFM image (Supplementary Information S3) and a nanogap measurement using a single cellulose nanofiber (Supplementary Information S4). Figure $3 \mathrm{c}$ shows the $I-V$ curves of the single cellulose nanofiber measurement. The series resistance formed by the AFM tip and the filament limits the current flow in the range of hundreds of nA. ${ }^{37}$ The measurement was performed at room temperature in atmospheric air conditions. After the forming process at a voltage of $0.3 \mathrm{~V}$, the cellulose nanofiber exhibited bipolar resistive switching. No significant difference in switching behavior was observed after 20 switching cycles, indicating the reliability of the switching of the single cellulose nanofiber. Figure 3d shows topographic AFM images before and after the resistive switching with various switching cycles. After the switching, the image commonly showed a decrease in the sample height. This is well consistent with the electrochemical Ag filament model $^{34,35}$ (see Supplementary Information S5) because a void should be created after the rupture of the Ag filament in the RESET process. Based on the Ag filament model, we analyzed the electrical conduction of the single cellulose nanofiber. Considering the resistivity of $\mathrm{Ag}$ $\left(1.59 \times 10^{-6} \Omega \mathrm{cm}\right)^{38}$ and the sample height of $15 \mathrm{~nm}$, the diameter of the Ag filament can be estimated to be ca. $12 \mathrm{~nm}$. This size is reasonable because the diameter of the cellulose nanofiber is ca. $15-20 \mathrm{~nm}^{36}$
Thus, these results highlight the excellent scalability of the nanopaper memory device down to the single cellulose nanofiber level.

Finally, we demonstrate the disposability of the nanopaper memory device. Before the disposal, the Ag electrode should be removed from the device because $\mathrm{Ag}$ has an antibacterial effect ${ }^{39,40}$ that suppresses the fungus-based biodegradation of the nanopaper in the natural environment. We found that the removal of the Ag electrode can be achieved by simply applying sonication in water (details are discussed in Supplementary Information S6). Next, we examined the biodegradability of the nanopaper memory device using natural soil. Our biodegradation-based disposal process using natural soil has some advantages with respect to the data security and the energy consumption compared with the techniques used in a previous work. ${ }^{31}$ The previously reported shredding process cannot be utilized when the device size becomes smaller than the blade spacing of the shredder, whereas the biodegradation process can be applied for any device scale. The burning process has high reliability for data destruction owing to the incineration of the device, but it consumes a high amount of energy to achieve the burning temperature. In contrast, the biodegradation of nanopaper may proceed at room temperature in natural soil, allowing us to reduce the energy consumption drastically. Figure 4a shows photographs of the nanopaper memory device after it is buried in natural soil. The details of the experiment can be seen in Supplementary Information S7. The temperature and the humidity of soil were controlled to be $22 \pm 2{ }^{\circ} \mathrm{C}$ and $92 \pm 3 \%$, respectively. As control experiments, nanopaper memory devices on $\mathrm{Si}$ and 
a

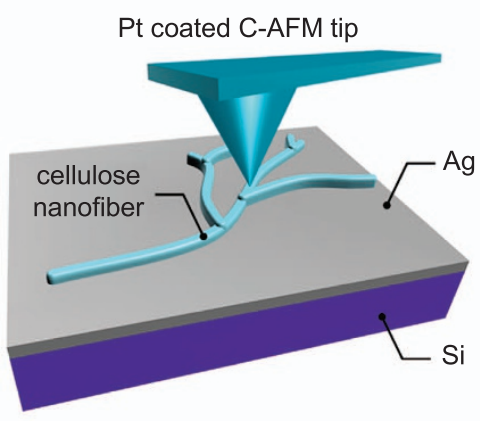

C

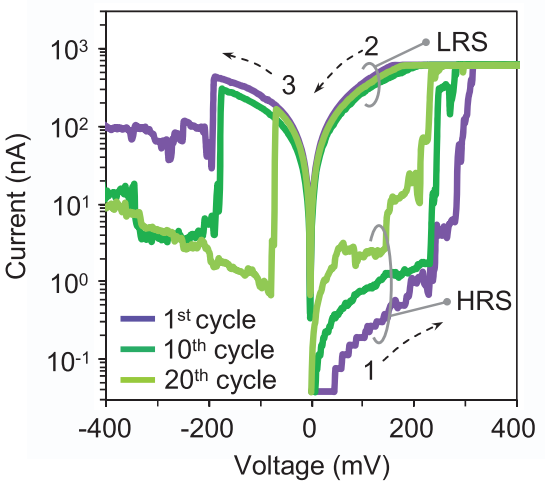

b

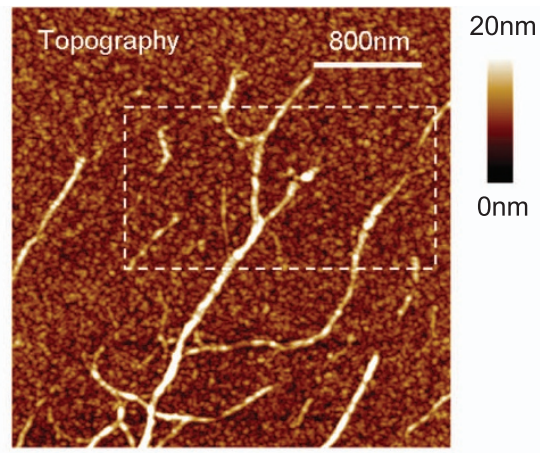

d

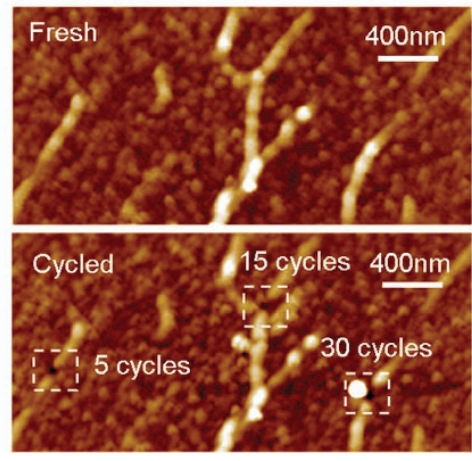

Figure 3 Examination of resistive switching using a single cellulose nanofiber. (a) Schematic illustration of the single cellulose nanofiber measurement system. To obtain the single cellulose nanofiber, the solution, created by the dilution of a 0.02 wt $\%$ cellulose nanofiber aqueous suspension $1: 1000$ with deionized (DI) water, was dropped onto the Ag-coated silicon substrate. The characterization of the resistive switching for the single cellulose nanofiber was performed by conductive-atomic force microscopy (C-AFM) with a Pt-coated Si cantilever. (b) Topographic AFM image of cellulose nanofibers on $\mathrm{Ag} / \mathrm{Si}$ substrate. The nanocellulose was raveled out to the single nanofiber level. (c) The current-voltage $(I-V)$ curves taken from a single cellulose nanofiber. The measurement was performed in atmospheric air conditions at room temperature. For the measurement, the voltage was applied to the Ag bottom electrode, and the Pt-coated cantilever was grounded. Similar to the resistive switching in the nanopaper memory, reversible resistive switching was seen in the single cellulose nanofiber. Similar switching characteristics were obtained after the 10th and 20th switching cycles. (d) Topographic AFM images of cellulose nanofiber before and after switching. Before the observation, the resistance switching was continued until the switching events diminished.

polyethylene terephthalate substrates were also examined. After 5 days, the nanopaper memory layer was no longer observed on all devices, whereas the substrate did not show any significant changes. The degradation of the nanopaper substrate started after 15 days and was complete after 26 days. Because the cellulose can be partially hydrolyzed in a high humidity environment and decomposed by microorganisms that have a cellulose-splitting enzyme, cellulase, the biodegradation of the nanopaper memory device might progress in natural soil. Similar biodegradation phenomena were reported for TEMPO (2,2,6,6-tetramethylpiperidine-1-oxyl)-oxidized nanopaper. ${ }^{41}$ On the other hand, the $\mathrm{Si}$ and polyethylene terephthalate substrates were stable even after 26 days, without showing degradation. To confirm the bacterial effect in natural soil, we conducted a biodegradation test by simply keeping our sample in room-air conditions (humidity 50-60\%), humid-air conditions (humidity 100\%) and water, as shown in Supplementary Information S8. In water, the resistive-switching layer was fully removed with the electrode in several minutes, whereas the nanopaper substrate was fully maintained after 35 days. On the other hand, in humid-air conditions and roomair conditions, both the resistive-switching layer and the nanopaper substrate partially (humid-air condition) and fully (room-air condition) remained after 35 days. We further examined the temperature effect in both the humid-air and water conditions. At $90^{\circ} \mathrm{C}$, there were no significant differences in the biodegradation effect compared with that in the same test performed at room temperature. This clearly indicates that the biodegradation of our nanopaper memory was caused by a bacterial effect in the natural soil. Figure $4 \mathrm{~b}$ shows the residual area ratio of the substrates after being buried in the soil. The degradation of the nanopaper substrate started after 12 days, whereas the $\mathrm{Si}$ and polyethylene terephthalate substrates fully remained after 26 days. These results indicate that we successfully demonstrated environment-friendly, disposable nonvolatile memory devices that are almost entirely composed of nanocellulose.

\section{CONCLUSIONS}

In this study, we demonstrate an environment-friendly, disposable nonvolatile memory device composed of 99.3 vol.\% nanocellulose. Our memory device consists of a nanocellulose-based resistive-switching layer and a nanopaper substrate. The device exhibited Ag filamentbased electrochemical redox nonvolatile resistive switching with the capability of multilevel storage and potential scalability down to the single nanofiber level (ca. $15 \mathrm{~nm}$ ). The biodegradability of our memory device was confirmed by burying it in natural soil for 26 days. These results indicate the potential of environment-friendly, disposable nonvolatile memory applications for developing a single-use monitoring device for health diagnosis. 

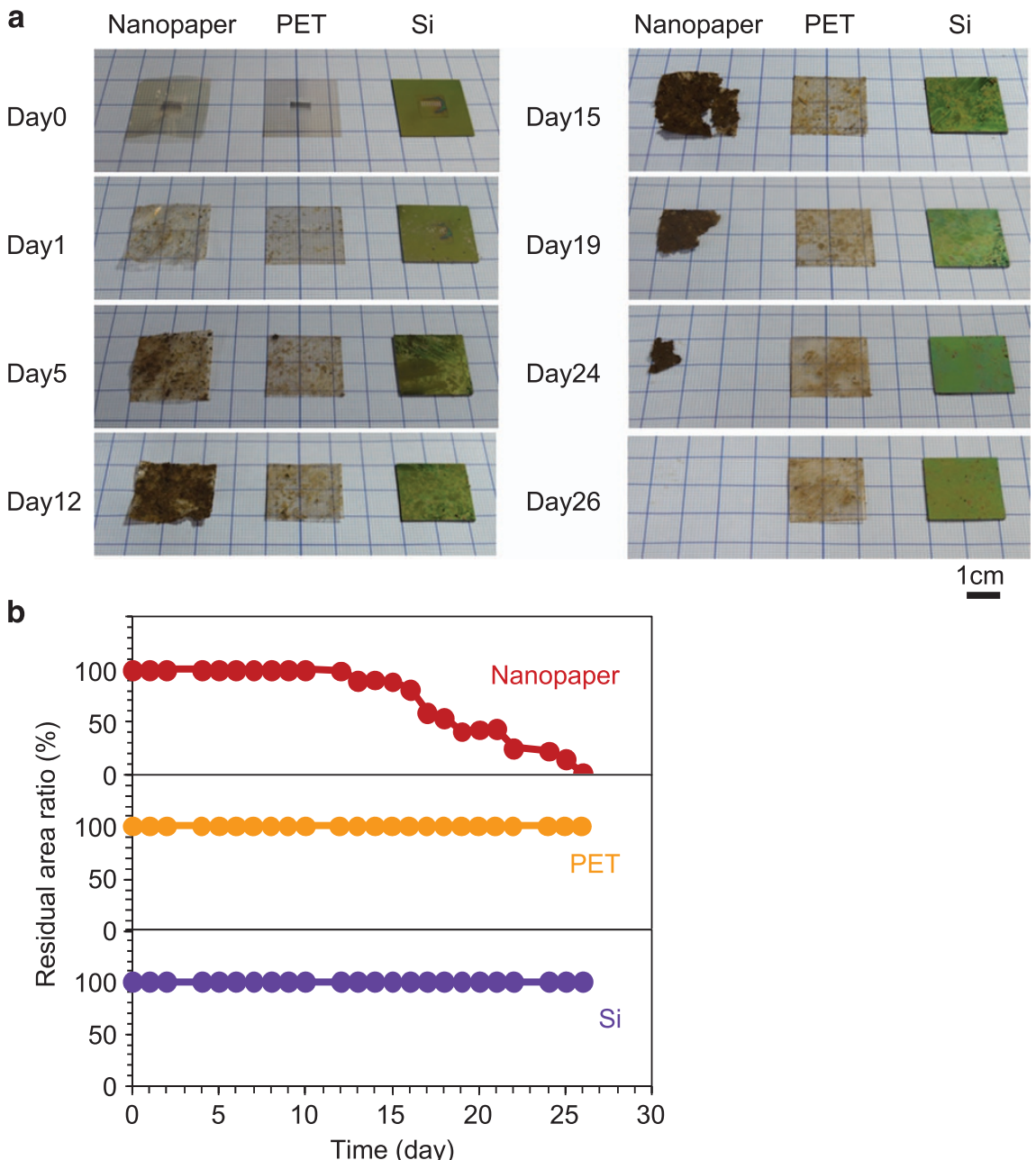

Figure 4 Biodegradability of the nanopaper memory device. (a) Time series of photographs for the nanopaper memory on the nanopaper substrate, polyethylene terephthalate (PET) substrate and Si substrate after burying in the natural soil. The test soil was collected in September at 34:49:30 north latitude and 135:31:28 east longitude. The soil ( $4 \mathrm{I}$ in total amount) was kept in a plastic bag to minimize the variation of the water concentration of the soil all through the biodegradation test. The humidity in the plastic bag was controlled to be ca. $90 \%$. (b) Time evolution of residual area ratio (\%) of nanopaper memory devices on nanopaper substrate (red), PET substrate (yellow) and Si substrate (purple). A significant decrease in the residual area ratio was obtained only for the nanopaper substrate, whereas the other substrates fully remained.

\section{CONFLICT OF INTEREST}

The authors declare no conflict of interest.

\section{ACKNOWLEDGEMENTS}

This study was supported by a Grant-in-Aid for Scientific Research (S) (No. 26220908). UC was supported by a PhD grant from the Agency for Innovation by Science and Technology (IWT).

Author contributions: UC, KN, HK, MN and TY conceived the nanopaper memory architecture. UC designed the device, performed the resistiveswitching measurements for both the nanopaper memory and the single cellulose nanofiber, analyzed the data and wrote the manuscript. KN conducted the single nanofiber measurement using the nanogap method, the Ag electrode removal experiment and the biodegradation experiment, analyzed the data and wrote the manuscript. FZ, GM and YH supported the biodegradation experiment. JDB, MJ and WV analyzed the data and revised the manuscript. HK, MN and TY supervised the project and revised the manuscript.

1 Ghoneim, M. T. \& Hussain, M. M. Review on physically flexible nonvolatile memory for internet of everything electronics. Electronics 4, 424-479 (2015).
2 Atzori, L., lera, A. \& Morabito, G. The internet of things: a survey. Comp. Netw. 54 , 2787-2805 (2010)

3 Miorandi, D., Sicari, S., Pellegrini, F. D. \& Chlamtac, I. Internet of things: vision, applications and research challenges. Ad Hoc Netw. 10, 1497-1516 (2012).

4 Suckling, J. \& Lee, J. Redefining scope: the true environmental impact of smartphones? Int. J. Life Cycle Assess. 20, 1181-1196 (2015).

5 Nogi, M., Iwamoto, S., Nakagaito, A. N. \& Yano, H. Optically transparent nanofiber paper. Adv. Mater. 21, 1595-1598 (2009).

6 Nogi, M. \& Yano, H. Optically transparent nanofiber sheets by deposition of transparent materials: a concept for a roll-to-roll processing. Appl. Phys. Lett. 94, 233117 (2009).

7 Nogi, M., Kim, C., Sugahara, T., Inui, T., Takahashi, T. \& Suganuma, K. High thermal stability of optical transparency in cellulose nanofiber paper. Appl. Phys. Lett. 102, 181911 (2013).

8 Yagyu, H., Saito, T., Isogai, A., Koga, H. \& Nogi, M. Chemical modification of cellulose nanofibers for the production of highly thermal resistant and optically transparent nanopaper for paper devices. ACS Appl. Mater. Interfaces 7, 22012-22017 (2015).

9 Huang, J., Zhu, H., Chen, Y., Preston, C., Rohrbach, K., Cumings, J. \& Hu, L. Highly transparent and flexible nanopaper transistors. ACS Nano 7, 2106-2113 (2013).

10 Yano, H., Sugiyama, J., Nakagaito, A. N., Nogi, M., Matsuura, T., Hikita, M. \& Handa, K. Optically transparent composites reinforced with networks of bacterial nanofibers. Adv. Mater. 17, 153-155 (2005).

11 Ho, M.-P., Wang, H., Lau, K.-T. \& Leng, J. Effect of silk fiber to the mechanical and thermal properties of its biodegradable composites. J. Appl. Polym. Sci. 127, 2389-2396 (2013).

12 Saravaman, D. Spider silk-structure, properties and spinning. J. Text. Apparel Technol. Manag. 5, 1-20 (2006) 
13 Hwang, S.-W., Tao, H., Kim, D.-H., Cheng, H., Song, J.-K., Rill, E., Brenckle, M. A., Panilaitis, B., Won, S. M., Kim, Y.-S., Song, Y. M., Yu, K. J., Ameen, A., Li, R., Su, Y., Yang, M., Kaplan, D. L., Zakin, M. R., Slepian, M. J., Huang, Y., Omenetto, F. G. \& Rogers, J. A. A physically transient form of silicon electronics. Science 337, 1640-1644 (2012).

14 Dagdeviren, C., Hwang, S.-W., Su, Y., Kim, S., Cheng, H., Gur, O., Haney, R. Omenetto, F. G., Huang, Y. \& Rogers, J. A. Transient, biocompatible electronics and energy harvesters based on ZnO. Small 20, 3398-3404 (2013).

15 Hwang, S.-W., Song, J.-K., Huang, X., Cheng, H., Kang, S.-K., Kim, B. H., Kim, J.-H., Yu, S., Huang, Y. \& Rogers, J. A. High-performance biodegradable/transient electronics on biodegradable polymers. Adv. Mater. 26, 3905-3911 (2014).

16 Kim, D.-H., Viventi, J., Amsden, J. J., Xiao, J., Vigeland, L., Kim, Y.-S., Blanco, J. A., Panilaitis, B., Frechette, E. S., Contreras, D., Kaplan, D. L., Omenetto, F. G., Huang, Y., Hwang, K.-C., Zakin, M. R., Litt, B. \& Rogers, J. A. Dissolvable films of silk fibroin for ultrathin conformal bio-integrated electronics. Nat. Mater. 9, 511-517 (2010).

17 Koga, H., Nogi, M., Komoda, N., Nge, T. T., Sugahara, T. \& Suganuma, K. Uniformly connected conductive networks on cellulose nanofiber paper for transparent paper electronics. NPG Asia Mater. 6, e93 (2014).

18 Nge, T. T., Nogi, M. \& Suganuma, K. Electrical functionality of inkjet-printed silver nanoparticle conductive tracks on nanostructured paper compared with those on plastic substrates. J. Mater. Chem. C 1, 5235-5247 (2013).

19 Toivonen, M. S., Kaskela, A., Rojas, O. J., Kauppinen, E. I. \& Ikkala, O. Ambient-dried cellulose nanofibril aerogel membranes with high tensile strength and their use for aerosol collection and templates for transparent, flexible devices. Adv. Funct. Mater. 25, 6618-6626 (2015).

20 Kang, W., Yan, C., Foo, C. Y. \& Lee, P. S. Foldable electrochromics enabled by nanopaper transfer method. Adv. Funct. Mater. 25, 4203-4210 (2015).

21 Fujisaki, Y., Koga, H., Nakajima, Y., Nakata, M., Tsuji, H., Yamamoto, T., Kurita, T., Nogi, M. \& Shimidzu, N. Transparent nanopaper-based flexible organic thin-film transistor array. Adv. Funct. Mater. 24, 1657-1663 (2014).

22 Jung, Y. H., Chang, T.-H., Zhang, H., Yao, C., Zheng, Q., Yang, V. W., Mi, H., Kim, M., Cho, S. J., Park, D.-W., Jiang, H., Lee, J., Quu, Y., Zhou, W., Cai, Z., Gong, S. \& Ma, Z. High-performance green flexible electronics based on biodegradable cellulose nanofibril paper. Nat. Commun. 6, 7170 (2015).

23 Zhu, H., Fang, Z., Preston, C., Li, Y. \& Hu, L. Transparent paper: fabrications, properties, and device applications. Energy Environ. Sci. 7, 269-287 (2014).

24 Hsieh, M.-C., Kim, C., Nogi, M. \& Suganuma, K. Electrically conductive lines on cellulose nanopaper for flexible electrical devices. Nanoscale 5, 9289-9295 (2013).

25 Fang, Z., Zhu, H., Yuan, Y., Ha, D., Zhu, S., Preston, C., Chen, Q., Li, Y., Han, X., Lee, S., Chen, G., Li, T., Munday, J., Huang, J. \& Hu, L. Novel nanostructured paper with ultrahigh transparency and ultrahigh haze for solar cells. Nano Lett. 14, 765-773 (2014)

26 Nogi, M., Karakawa, M., Komoda, N., Yagyu, H. \& Nge, T. T. Transparent Conductive Nanofiber Paper for Foldable Solar Cells. Sci. Rep. 5, 17254 (2015).

27 Inui, T., Koga, H., Nogi, M., Komoda, N. \& Suganuma, K. A miniaturized flexible antenna printed on a high dielectric constant nanopaper composite. Adv. Mater. 27, 1112-1116 (2015).

28 Nogi, M., Komoda, N., Otsukac, K. \& Suganuma, K. Foldable nanopaper antennas for origami electronics. Nanoscale 5, 4395-4399 (2013).

29 Zhong, J., Zhu, H., Zhong, Q., Dai, J., Li, W., Jang, S.-H., Yao, Y., Henderson, D. $\mathrm{Hu}, \mathrm{Q}$., Hu, L. \& Zhou, J. Self-powered human-interactive transparent nanopaper systems. ACS Nano 9, 7399-7406 (2015).
30 Nagashima, K., Koga, H., Celano, U., Zhuge, F. W., Kanai, M., Rahong, S., Meng, G., He, Y., Boeck, J. D., Jurczak, M., Vandervorst, W., Kitaoka, T., Nogi, M. \& Yanagida, T. Cellulose nanofiber paper as an ultra flexible nonvolatile memory. Sci. Rep. 4, 5532 (2014).

31 Lien, D.-H., Kao, Z.-K., Huang, T.-H., Liao, Y.-C., Lee, S.-C. \& He, J.-H. All-Printed Paper Memory. ACS Nano 8, 7613-7619 (2014).

32 Celano, U., Goux, L., Belmonte, A., Opsomer, K., Franquet, A., Schulze, A., Detavernier, C., Richard, O., Bender, H., Jurczak, M. \& Vandervorst, W. Threedimensional observation of the conductive filament in nanoscaled resistive memory devices. Nano Lett. 14, 2401-2406 (2014).

33 Yanagida, T., Nagashima, K., Oka, K., Kanai, M., Klamchuen, A., Park, B. H. \& Kawai, T. Scaling effect on unipolar and bipolar resistive switching of metal oxides. Sci. Rep. 3, 1657 (2013).

34 Yang, Y., Gao, P., Gaba, S., Chang, T., Pan, X. \& Lu, W. Observation of conducting filament growth in nanoscale resistive memories. Nat. Commun. 3, 732 (2012)

35 Valov, I., Linn, E., Tappertzhofen, S., Schmelzer, S., Hurk, J., Lentz, F. \& Waser, R. Nanobatteries in redox-based resistive switches require extension of memristor theory. Nat. Commun. 4, 1771 (2013).

36 Uetani, K. \& Yano, H. Nanofibrillation of wood pulp using a high-speed blender. Biomacromolecules 12, 348-353 (2011).

37 Celano, U., Goux, L., Opsomer, K., Belmonte, A., lapichino, M., Detavernier, C., Jurczak, M. \& Vandervorst, W. Switching mechanism and reverse engineering of low-power Cu-based resistive switching devices. Nanoscale 5, 11187-11192 (2013).

38 Serway, R. A. Principles of Physics (Saunders College Pub., London, Fort Worth, Texas, 1998).

39 Davoudi, Z. M., Kandjani, A. E., Bhatt, A. I., Kyratzis, I. L., O'Mullane, A. P. \& Bansal, V. Hybrid antibacterial fabrics with extremely high aspect ratio Ag/AgTCNQ nanowires. Adv. Funct. Mater. 24, 1047-1053 (2014).

40 Fellahi, O., Sarma, R. K., Das, M. R., Saikia, R., Marcon, L., Coffinier, Y., Hadjersi, T., Maamache, M. \& Boukherroub, R. The antimicrobial effect of silicon nanowires decorated with silver and copper nanoparticles. Nanotechnology 24, 495101 (2013).

41 Homma, I., Fukuzumi, H., Saito, T. \& Isogai, A. Effects of carboxyl-group counter-ions on biodegradation behaviors of TEMPO-oxidized cellulose fibers and nanofibril films. Cellulose 20, 2505-2515 (2013).

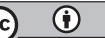

This work is licensed under a Creative Commons Attribution 4.0 International License. The images or other third party material in this article are included in the article's Creative Commons license, unless indicated otherwise in the credit line; if the material is not included under the Creative Commons license, users will need to obtain permission from the license holder to reproduce the material. To view a copy of this license, visit http:// creativecommons.org/licenses/by/4.0/

(C) The Author(s) 2016

Supplementary Information accompanies the paper on the NPG Asia Materials website (http://www.nature.com/am) 